\title{
Effective temperatures of red giants in the APOKASC catalogue and the mixing length calibration in stellar models
}

\author{
M. Salaris ${ }^{1}$, S. Cassisi ${ }^{2}$, R. P. Schiavon ${ }^{1}$, and A. Pietrinferni ${ }^{2}$ \\ ${ }^{1}$ Astrophysics Research Institute, Liverpool John Moores University, IC2 Building, Liverpool Science Park, 146 Brownlow Hill, \\ Liverpool L3 5RF, UK \\ e-mail: M.Salaris@ljmu.ac.uk \\ 2 INAF-Osservatorio Astronomico d'Abruzzo, via M. Maggini, sn, 64100 Teramo, Italy
}

Received 22 November 2017 / Accepted 18 January 2018

\begin{abstract}
Red giants in the updated APOGEE-Kepler catalogue, with estimates of mass, chemical composition, surface gravity and effective temperature, have recently challenged stellar models computed under the standard assumption of solar calibrated mixing length. In this work, we critically reanalyse this sample of red giants, adopting our own stellar model calculations. Contrary to previous results, we find that the disagreement between the $T_{\text {eff }}$ scale of red giants and models with solar calibrated mixing length disappears when considering our models and the APOGEE-Kepler stars with scaled solar metal distribution. However, a discrepancy shows up when $\alpha$-enhanced stars are included in the sample. We have found that assuming mass, chemical composition and effective temperature scale of the APOGEE-Kepler catalogue, stellar models generally underpredict the change of temperature of red giants caused by $\alpha$-element enhancements at fixed $[\mathrm{Fe} / \mathrm{H}]$. A second important conclusion is that the choice of the outer boundary conditions employed in model calculations is critical. Effective temperature differences (metallicity dependent) between models with solar calibrated mixing length and observations appear for some choices of the boundary conditions, but this is not a general result.
\end{abstract}

Key words. convection - stars: low mass - stars: fundamental parameters

\section{Introduction}

Calculations of the superadiabatic convective temperature gradients in stellar evolution models are almost universally based on the very simple, local formalism provided by the mixing length theory (MLT; Böhm-Vitense 1958). The convective flow is idealized in terms of columns of upwards and downwards moving elements all with the same characteristic size, that cover a fixed mean free path before dissolving. Both the mean free path and the characteristic size of the convective elements are assumed to be equal to $\Lambda=\alpha_{\mathrm{MLT}} H_{P}$, the mixing length. The free parameter $\alpha_{\text {MLT }}$ is typically assumed to be a constant value within the convective regions and along all evolutionary phases, and $H_{p}$ is the local pressure scale height. The chosen value of $\alpha_{\mathrm{MLT}}$ determines the model $T_{\text {eff. }}$.

It is well known that this simplistic MLT picture of convection is very different from results of two-dimensional (2D) and three-dimensional (3D) radiation hydrodynamics simulations of convection in stellar envelopes and atmospheres (see, e.g. Stein \& Nordlund 1989; Ludwig et al. 1999; Trampedach et al. 2014; Magic et al. 2015, and references therein). These computations show how convection consists mainly of continuous flows, with the warm gas rising almost adiabatically, in a background of cool, narrower and faster downdrafts. A fraction of the upflows is continuously overturning to conserve mass on the background of the steep density gradients.

Clearly, we cannot expect the MLT to provide an accurate description of the thermal stratification within the superadiabatic layers of convective envelopes, but only an effective stratification that leads hopefully to an appropriate effective temperature ( $\left.T_{\text {eff }}\right)$ scale for the stellar models, once a suitable value of $\alpha_{\text {MLT }}$ is chosen. This free parameter is usually calibrated by reproducing the radius of the Sun at the solar age with an evolutionary solar model (Gough \& Weiss 1976). Of course there is no reason a priori why $\alpha_{\text {MLT }}$ should be the same with varying mass, evolutionary phase, and chemical composition.

Additional free parameters appear in the MLT formalism, but they are generally fixed beforehand, giving origin to different flavours of the MLT formalism (see, e.g. Pedersen et al. 1990; Salaris \& Cassisi 2008, and references therein). Remarkably, different MLT flavours found in the literature provide essentially the same evolutionary tracks when $\alpha_{\mathrm{MLT}}$ is accordingly recalibrated on the Sun (Pedersen et al. 1990; Salaris \& Cassisi 2008).

One independent empirical way to calibrate and/or test whether the solar calibration of $\alpha_{\mathrm{MLT}}$ is appropriate also for other evolutionary phases/chemical compositions, is to compare empirically determined effective temperatures of red giant branch (RGB) stars, with theoretical models of the appropriate chemical composition, that are indeed very sensitive to the treatment of the superadiabatic layers (see, e.g. Straniero \& Chieffi 1991; Salaris \& Cassisi 1996; VandenBerg et al. 1996, and references therein).

A very recent study by Tayar et al. (2017) has analysed a sample of over 3000 RGB stars with $T_{\text {eff }}$, mass, surface gravity $(g)$, $[\mathrm{Fe} / \mathrm{H}]$ and $[\alpha / \mathrm{Fe}]$ determinations from the updated APOGEEKepler catalogue (APOKASC), suited for testing the mixing length calibration in theoretical stellar models. According to the grid of stellar evolution models specifically calculated to match the $T_{\text {eff }}$ values of the individual stars, this study (hereafter T17) concluded that a variation of $\alpha_{\text {MLT }}$ with varying [Fe/H] is required. Their stellar models with solar calibrated $\alpha_{\mathrm{MLT}}$ (the solar $\alpha_{\mathrm{MLT}}$ in their calculations is equal to 1.72 when employing 
the Böhm-Vitense 1958, MLT flavour) are unable to match the empirical $T_{\text {eff }}$ values for $[\mathrm{Fe} / \mathrm{H}]$ between $\sim+0.4$ and -1.0 . They calculated differences $\Delta T \equiv T_{\text {obs }}-T_{\text {models }}$ between observed and theoretical $T_{\text {eff }}$ for each individual star in their sample, and found that $\Delta T=93.1[\mathrm{Fe} / \mathrm{H}]+107.5 \mathrm{~K}$. They concluded that a varying mixing length $\alpha_{\mathrm{MLT}}=0.161[\mathrm{Fe} / \mathrm{H}]+1.90$ is required to match the empirical temperatures. This relationship predicts a non solar $\alpha_{\text {MLT }}$ also for RGB stars at solar metallicity. The same authors found a similar trend of $\Delta T$ with $[\mathrm{Fe} / \mathrm{H}]$ (a $\Delta T-[\mathrm{Fe} / \mathrm{H}]$ slope of about $100 \mathrm{~K} \mathrm{dex}^{-1}$ ) when using the PARSEC stellar models (Bressan et al. 2012), albeit with a zero point offset of about $-100 \mathrm{~K}$ compared to the results obtained with their models.

A variation of $\alpha_{\text {MLT }}$ with [Fe/H] - and potentially with evolutionary phase - has obviously profound impl ications for the calibration of convection in stellar models, age estimates of RGB stars in the Hertzsprung-Russell and $g-T_{\text {eff }}$ diagrams, and also stellar population integrated spectral features sensitive to the presence of a RGB component.

In light of the relevant implications of T17 result, we have reanalysed their APOKASC sample with our own independent stellar evolution calculations, paying particular attention to the role played by uncertanties in the calculation of the model boundary conditions. Our new results clarify the role played by the combination of $\alpha_{\mathrm{MLT}}$ and boundary conditions in the interpretations of the data, and, very importantly, disclose also a major difficulty when comparing models with T17 $\alpha$-enhanced stars.

Section 2 briefly summarizes T17 data and the models calculated for this work, followed in Sect. 3 by a description of our analysis and our new results. A summary and in-depth discussion of our findings closes the paper.

\section{Models and data}

For our analysis we have calculated a large set of models using code and physics inputs employed to create the BaSTI database of stellar evolution models (see Pietrinferni et al. 2004). Because of the relevance to this work, we specify that radiative opacities are from the OPAL calculations (Iglesias \& Rogers 1996) for temperatures larger than $\log (T)=4.0$, whereas calculations by Ferguson et al. (2005) - that include the contributions from molecules and grains - have been adopted for lower temperatures. Both high- and low-temperature opacity tables account properly for the metal distributions adopted in our models (see below and Sect. 3.2).

We have just changed the $T(\tau)$ relation adopted in BaSTI to determine the models' outer boundary conditions (a crucial input for the determination of the models' $T_{\text {eff }}$, as discussed in Sect. 3.3), employing the Vernazza et al. (1981) solar semiempirical $T(\tau)$ (hereafter VAL) instead of the Krishna Swamy (1966) one ${ }^{1}$. According to the analysis by Salaris \& Cassisi (2015), model tracks computed with this $T(\tau)$ relation approximate well results obtained using the hydro-calibrated $T(\tau)$ relationships provided by Trampedach et al. (2014) for the solar chemical composition. We will come back to this issue in Sect. 3.3.

We have computed a model grid for masses between 0.7 and $2.6 M_{\odot}$ in $0.1 M_{\odot}$ increments, and scaled solar $[\mathrm{Fe} / \mathrm{H}]$ between -2.0 and +0.4 dex in steps of $0.2-0.3$ dex. A solar model including atomic diffusion has been calibrated to determine initial solar

\footnotetext{
1 We implement the following fit to Vernazza et al. (1981) tabulation: $T^{4}=0.75 T_{\text {eff }}^{4}\left(\tau+1.017-0.3 e^{-2.54 \tau}-0.291 e^{-30 \tau}\right)$, where $\tau$ is the Rosseland optical depth.
}
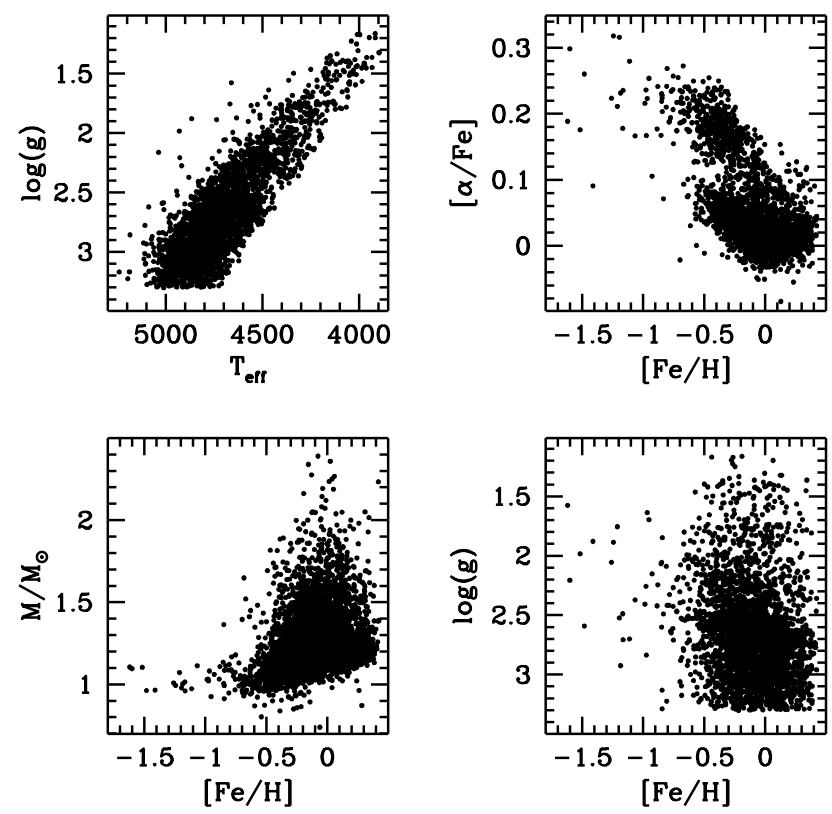

Fig. 1. The adopted sample of RGB stars from the APOKASC dataset displayed in various diagrams: $\log (g)-T_{\mathrm{eff}},[\alpha / \mathrm{Fe}]-[\mathrm{Fe} / \mathrm{H}]$, mass $-[\mathrm{Fe} / \mathrm{H}]$ and $\log (g)-[\mathrm{Fe} / \mathrm{H}]$.

values of He and metal mass fractions $Y=0.274, Z=0.0199$ (for the Grevesse \& Noels 1993, solar metal mixture), and mixing length (with the MLT flavour from Böhm-Vitense 1958) $\alpha_{\text {MLT }}=1.90$. We have adopted the same $Y-Z$ relationship $Y=$ $0.245+1.41 \times Z$ as in BaSTI. Our model grid does not include atomic diffusion, because its effect on the RGB evolution is, as well known, negligible (Salaris \& Cassisi 2015). In addition, we have calculated sets of $\alpha$-enhanced models for the various masses and $[\mathrm{Fe} / \mathrm{H}]$ of the grid, and $[\alpha / \mathrm{Fe}]=0.4$.

With these models we have first reanalysed the sample of RGB field stars by T17, considering the $\log (g), T_{\text {eff }},[\mathrm{Fe} / \mathrm{H}]$ and $[\alpha / \mathrm{Fe}]$ values listed in the publicly available data file. Masses are derived from asteroseismic scaling relations, and the other quantities are obtained using the APOGEE spectroscopic data set. The $T_{\text {eff }}$ values are calibrated to be consistent with the González Hernández \& Bonifacio (2009) effective temperature scale, based on the infrared-flux method.

We considered only the stars with a calculated error bar on the mass determinations (we excluded objects with error on the mass given as -9999), that still leave a sample of well over 3000 objects, spanning a mass range between 0.8 and $2.4 M_{\odot}$, with a strong peak of the mass distribution around 1.2-1.3 $M_{\odot}$.

Figure 1 displays the data in four different diagrams. The stars cover a $\log (g)$ range between $\sim 3.3$ and 1.1 (in cgs units), and $T_{\text {eff }}$ between $\sim 5200$ and $3900 \mathrm{~K}$, with the bulk of the stars having $[\mathrm{Fe} / \mathrm{H}]$ between $\sim-0.7$ and $\sim+0.4 \mathrm{dex}$, and a maximum $\alpha$-enhancement typically around 0.25 dex. Notice that stars with a given $[\mathrm{Fe} / \mathrm{H}]$ typically cover the full range of surface gravities, but the range of masses at a given $[\mathrm{Fe} / \mathrm{H}]$ varies with metallicity, due to the variation of the age distribution of Galactic disk stars with $[\mathrm{Fe} / \mathrm{H}]$.

To determine differences $\Delta T \equiv T_{\text {obs }}-T_{\text {models }}$ between observed and theoretical $T_{\text {eff }}$ for each individual star in T17 sample, we have interpolated linearly in mass, $[\mathrm{Fe} / \mathrm{H}],[\alpha / \mathrm{Fe}], \log (g)$ amongst the models, to determine the corresponding theoretical $T_{\text {eff }}$ for each observed star. 
Table 1. Mean values of the differences $\Delta T$ for the full $T 17$ sample as a function of $[\mathrm{Fe} / \mathrm{H}]$.

\begin{tabular}{rccc}
\hline \hline$[\mathrm{Fe} / \mathrm{H}]$ & $\pm \Delta[\mathrm{Fe} / \mathrm{H}]$ & $\langle\Delta T\rangle(\mathrm{K})$ & $\sigma(\Delta T)(\mathrm{K})$ \\
\hline 0.35 & 0.05 & -35 & 24 \\
0.25 & 0.05 & -23 & 33 \\
0.15 & 0.05 & -21 & 31 \\
0.05 & 0.05 & -8 & 35 \\
-0.05 & 0.05 & -11 & 40 \\
-0.15 & 0.05 & -9 & 44 \\
-0.25 & 0.05 & -22 & 52 \\
-0.35 & 0.05 & -32 & 54 \\
-0.45 & 0.05 & -54 & 53 \\
-0.60 & 0.10 & -72 & 55
\end{tabular}

Notes. The mean values of $\Delta T$ (third column) are determined in $[\mathrm{Fe} / \mathrm{H}]$ bins centred around the values given in the first column, and half-widths listed in the second column. The last column displays the $1 \sigma$ dispersion around the mean $\Delta T$.

\section{Analysis of T17 sample}

The bottom panel of Fig. 2 displays the differences $\Delta T \equiv$ $T_{\text {obs }}-T_{\text {models }}$ as a function of $[\mathrm{Fe} / \mathrm{H}]$ for the full $\mathrm{T} 17$ sample; we have considered stars with $[\mathrm{Fe} / \mathrm{H}]>-0.7$, to include only the $[\mathrm{Fe} / \mathrm{H}]$ range well sampled by the data. It is easy to notice a trend of $\Delta T$ with $[\mathrm{Fe} / \mathrm{H}]$, qualitatively similar to what found by $\mathrm{T} 17$. We have overplotted, as open circles, mean values of $\Delta T$ determined in ten $[\mathrm{Fe} / \mathrm{H}]$ bins, with total width of 0.10 dex, apart from the most metal poor bin, that has a width of 0.20 dex, due to the smaller number of stars populating that metallicity range - see Table 1. The horizontal error bars cover the width of the individual bins, while the vertical ones denote the $1 \sigma$ dispersion of $\Delta T$ around the mean values.

As in T17, we find a drop of $\Delta T$ with decreasing $[\mathrm{Fe} / \mathrm{H}]$, when $[\mathrm{Fe} / \mathrm{H}]$ is below $\sim-0.25$. If we perform a simple linear fit through these mean values (considering the $1 \sigma$ dispersion as the error on these mean $\Delta T$ values) we obtain for the full sample $\Delta T=(39 \pm 19)[\mathrm{Fe} / \mathrm{H}]-(25 \pm 6) \mathrm{K}$, valid over a $[\mathrm{Fe} / \mathrm{H}]$ range of $\sim 1.1$ dex. A linear fit is clearly not the best approximation of the $\Delta T-[\mathrm{Fe} / \mathrm{H}]$ global trend - as mentioned also in $\mathrm{T} 17-$ but it replicates T17 analysis and suffices to highlight the main results of these comparisons.

Our RGB models turn out to be systematically hotter than observations by just $25 \mathrm{~K}$ at solar $[\mathrm{Fe} / \mathrm{H}]$, a negligible value considering the error on the González Hernández \& Bonifacio (2009) $T_{\text {eff }}$ calibration (the quoted average error on their RGB $T_{\text {eff }}$ scale is $\leq 76 \mathrm{~K}$ ), but the differences increase with decreasing $[\mathrm{Fe} / \mathrm{H}]$. The slope we derive is about half the value of the slope determined by $\mathrm{T} 17$ with their own calculations, and the zero point is about $130 \mathrm{~K}$ lower. We do not find any correlation between $\Delta T$ and the surface gravity $g$ of the observed stars, as shown in Fig. 3.

The top panel of Fig. 2 displays $\Delta T$ values considering this time only stars with essentially scaled solar metal mixture, that is $[\alpha / \mathrm{Fe}]<0.07$ (we chose this upper limit that is approximately equal to five times the $1 \sigma$ error on $[\alpha / \mathrm{Fe}]$ quoted in T17 data, but an upper limit closer to zero does not change the results). The overplotted mean values in the various $[\mathrm{Fe} / \mathrm{H}]$ bins are reported in Table 2. This time the trend of $\Delta T$ with $[\mathrm{Fe} / \mathrm{H}]$ is not statistically significant. A linear fit to the mean $\Delta T$ values provides $\Delta T=(-9 \pm 15)[\mathrm{Fe} / \mathrm{H}]-(14 \pm 5)$, meaning that now theory and observations are essentially in agreement.
Table 2. As Table 1, but for stars with observed $[\alpha / \mathrm{Fe}]<0.07$.

\begin{tabular}{rccc}
\hline \hline$[\mathrm{Fe} / \mathrm{H}]$ & $\pm \Delta[\mathrm{Fe} / \mathrm{H}]$ & $\langle\Delta T\rangle(\mathrm{K})$ & $\sigma(\Delta T)(\mathrm{K})$ \\
\hline 0.35 & 0.05 & -34 & 23 \\
0.25 & 0.05 & -22 & 30 \\
0.15 & 0.05 & -18 & 29 \\
0.05 & 0.05 & -4 & 32 \\
-0.05 & 0.05 & -4 & 37 \\
-0.15 & 0.05 & 0 & 39 \\
-0.25 & 0.05 & 0 & 39 \\
-0.35 & 0.05 & -7 & 38 \\
-0.45 & 0.05 & -7 & 43 \\
-0.60 & 0.10 & -34 & 52 \\
\hline
\end{tabular}

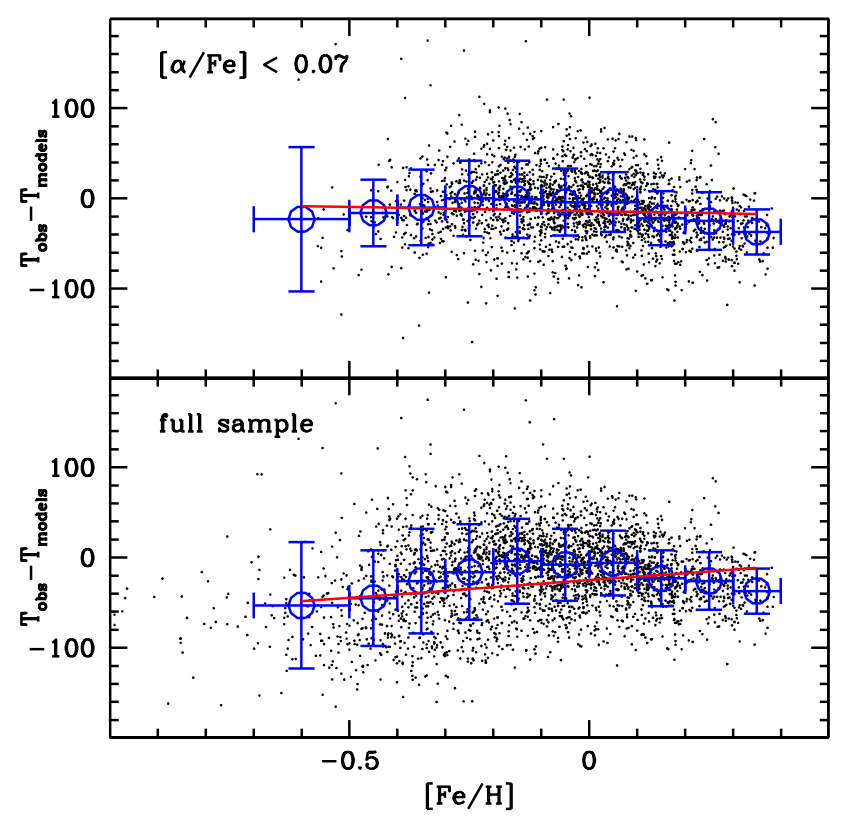

Fig. 2. $\Delta T$ as a function of $[\mathrm{Fe} / \mathrm{H}]$ (dots) for the whole sample of RGB stars (bottom panel) and for a sub-sample of RGB objects with $[\alpha / \mathrm{Fe}]<0.07$ (top panel). Open circles with error bars denote the mean values of $\Delta T$ in specific metallicity bins - Cols. 3 and 4 in Tables 1 and 2 - while the solid lines display linear fits to the binned data.

Figure 4 makes clearer the reason for the different result obtained when neglecting the $\alpha$-enhanced stars. We show here histograms of $\Delta T$ values for stars with $[\mathrm{Fe} / \mathrm{H}]$ between -0.5 and $-0.3,[\alpha / \mathrm{Fe}]<0.07$ and $[\alpha / \mathrm{Fe}] \geq 0.07$, respectively. One can clearly see how, in the same $[\mathrm{Fe} / \mathrm{H}]$ range, we determine for $\alpha$-enhanced stars systematically lower $\Delta T$ values.

In conclusion, with our calculations the trend of $\Delta T$ with $[\mathrm{Fe} / \mathrm{H}]$ is introduced by the inability of $\alpha$-enhanced models with solar calibrated $\alpha_{\text {MLT }}$ to match their observational counteparts, that is stellar models are increasingly hotter than observations when $[\alpha / \mathrm{Fe}]$ increases, at fixed $[\mathrm{Fe} / \mathrm{H}]$, even though we have taken into account the theoretically expected effect of $[\alpha / \mathrm{Fe}]$ on the model $T_{\text {eff }}$, at a given $[\mathrm{Fe} / \mathrm{H}]$. Comparing the values in Tables 1 and 2 one can notice that the effect of excluding $\alpha$-enhanced stars on the mean $\Delta T$ values appears around $[\mathrm{Fe} / \mathrm{H}]=-0.25$, consistent with the fact that below this $[\mathrm{Fe} / \mathrm{H}]$ the fraction of $\alpha$-enhanced stars increases, and $[\alpha / \mathrm{Fe}]$ values also increase. On the other hand, and very importantly, our models with solar calibrated $\alpha_{\text {MLT }}$ produce $T_{\text {eff }}$ values for scaled solar stars that are generally consistent with observations over a $[\mathrm{Fe} / \mathrm{H}]$ range of about $1 \mathrm{dex}$. 


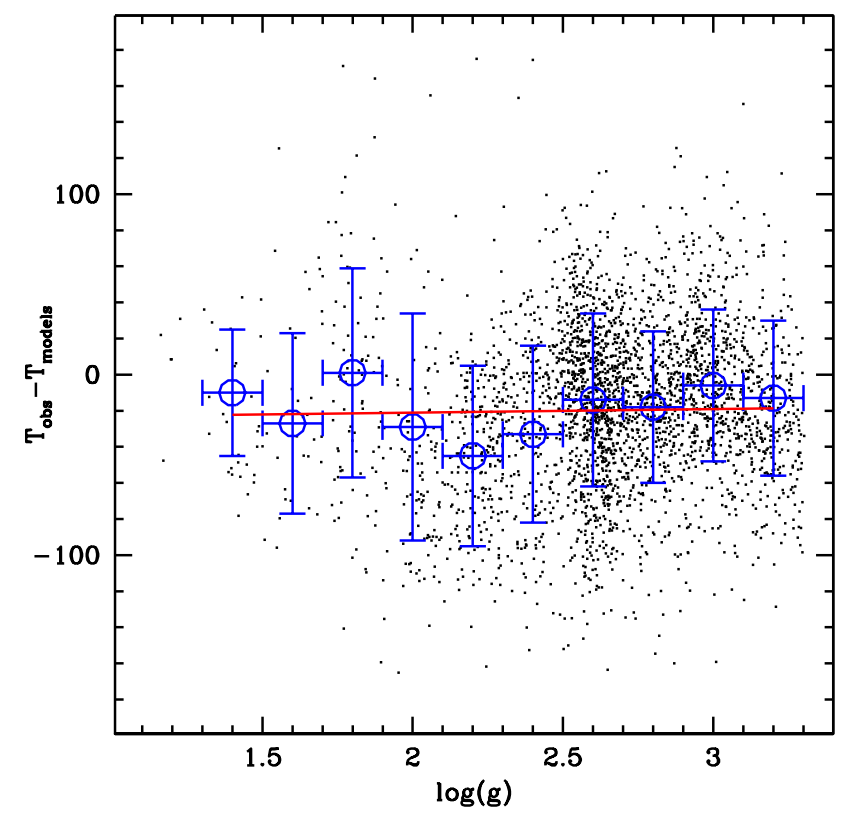

Fig. 3. $\Delta T$ as a function of $\log (g)$ for the whole sample of RGB stars. Open circles with error bars denote the mean values of $\Delta T$ in 0.2 dex $\log (g)$ bins, and the $1 \sigma$ dispersion around these mean values. The solid line displays a linear fit to the binned data, with a slope that is consistent with zero (the slope is equal to $2 \pm 8 \mathrm{~K} / \mathrm{dex}$ ).

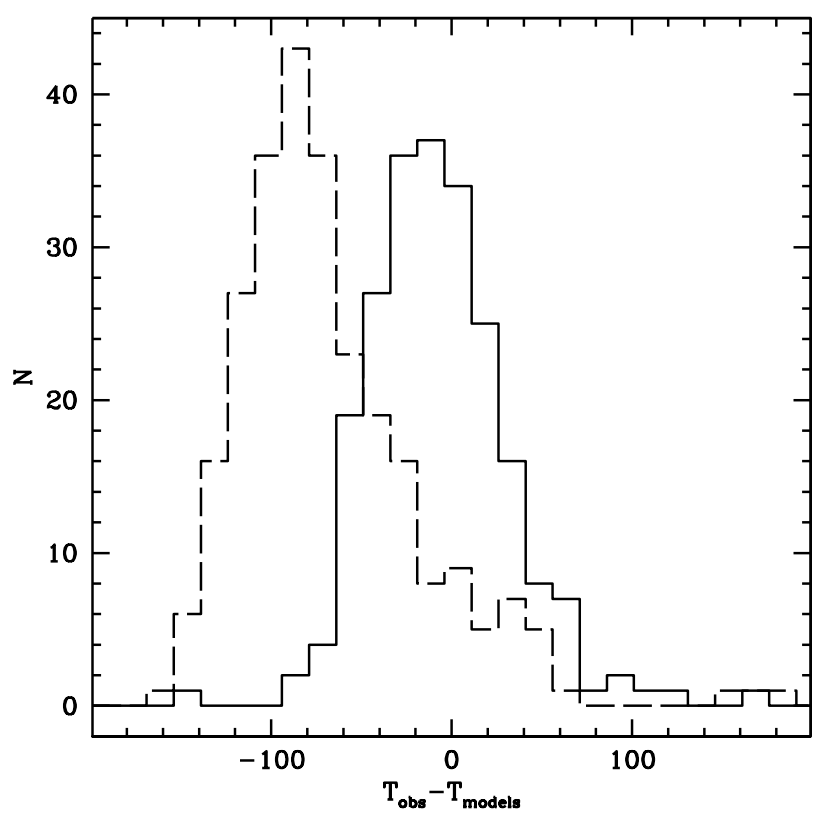

Fig. 4. Histograms $\Delta T$ for stars with $[\mathrm{Fe} / \mathrm{H}]$ between -0.5 and -0.3 , $[\alpha / \mathrm{Fe}]<0.07$ (solid line) and $[\alpha / \mathrm{Fe}] \geq 0.07$ (dashed line)

Our conclusions appear to be very different from T17 results obtained with their own model calculations, and therefore we have reanalysed T17 temperature differences (with respect to their own solar calibrated $\alpha_{\text {MLT }}$ models) on a star-by-star basis as provided by the authors ${ }^{2}$, using exactly the same $[\mathrm{Fe} / \mathrm{H}]$ bins discussed before. The results are displayed in Fig. 5. T17 $\Delta T$ values have been binned in the same $[\mathrm{Fe} / \mathrm{H}]$ ranges employed for our own results, and we have then performed a linear fit to the mean

2 T17 did not provide the $\Delta T$ values they obtained employing the PARSEC models.

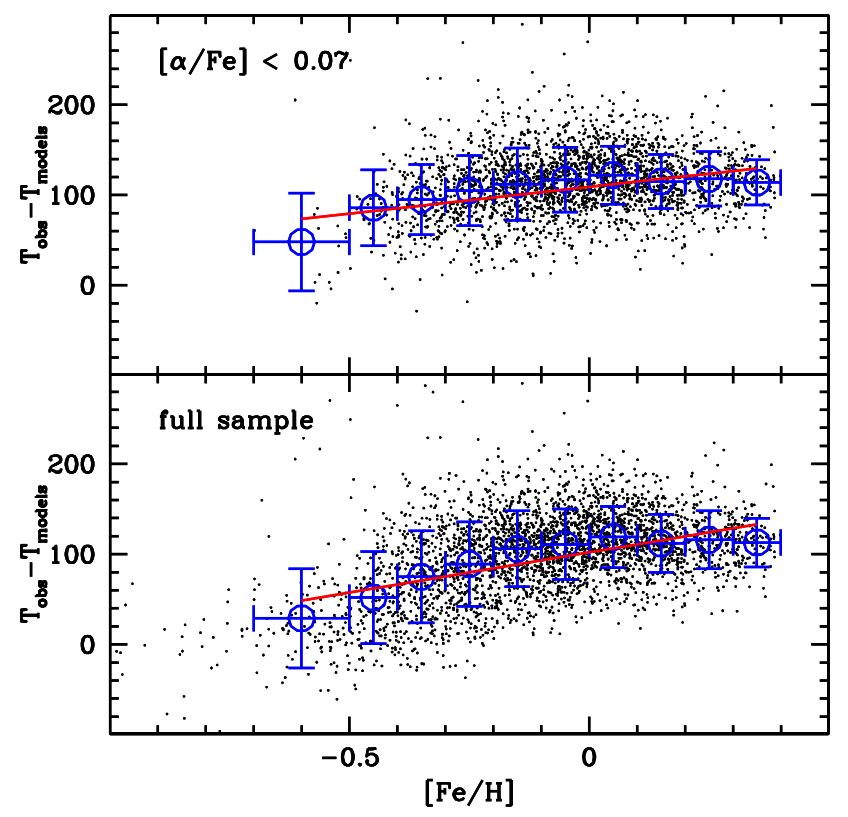

Fig. 5. As Fig. 2 but in this case individual $\Delta T$ values are from the study by $\mathrm{T} 17$.

values of the ten $[\mathrm{Fe} / \mathrm{H}]$ bins for both the full sample, and the sample restricted to stars with $[\alpha / \mathrm{Fe}]<0.07$.

The linear fit to the full sample provides $\Delta T=$ $(89 \pm 16)[\mathrm{Fe} / \mathrm{H}]+(102 \pm 5) \mathrm{K}$. Slope and zero point are well consistent with the values $(93.1 \mathrm{~K} / \mathrm{dex}$ for the slope and $107.5 \mathrm{~K}$ for the zero point) determined by T17 using their different finer - binning of the data. This means that the slight different way to analyse $\Delta T$ values as employed in our analysis, provides exactly the same results found by T17, when applied to T17 estimates of $\Delta T$.

Restricting the sample to objects with $[\alpha / \mathrm{Fe}]<0.07$, the linear fit to T17 $\Delta T$ values provides $\Delta T=(59 \pm 14)[\mathrm{Fe} / \mathrm{H}]+$ $(109 \pm 5) \mathrm{K}$. A slope different from zero is still present, contrary to what we find with our calculations, hence it cannot be attributed to just an inconsistent modelling of the $\alpha$-enhanced population. On the other hand, this slope is lower than the case of the full sample, and implies that the match of $\alpha$-enhanced stars with solar $\alpha_{\text {MLT }}$ models increases the trend of $\Delta T$ with $[\mathrm{Fe} / \mathrm{H}]$, compared to the case of just stars with scaled solar metal composition.

This is exemplified by Fig. 6, that is the same as Fig. 4, this time considering T17 $\Delta T$ values. One can see clearly that also in case of T17 models, $\alpha$-enhanced stars at the same $[\mathrm{Fe} / \mathrm{H}]$ display different (lower) $\Delta T$ compared to the scaled solar counterparts, exactly as in case of our models.

It is also important to notice also a large difference, of about $120 \mathrm{~K}$, in the zero points compared to our results.

\subsection{Revisiting the chemical composition of T17 stars}

In light of the inconsistency between our solar calibrated $\alpha_{\text {MLT }}$ models for $\alpha$-enhanced compositions and the observed $\alpha$-enhanced stars, we have investigated in more detail the chemical composition of T17 sample, looking at the abundances reported in the APOGEE DR13 catalogue (Majewski et al. 2017 and Holtzman et al., in prep.).

We have realized that $[\mathrm{Fe} / \mathrm{H}]$ values reported by $\mathrm{T} 17$ are actually labelled as $[\mathrm{M} / \mathrm{H}]$ in the DR13 catalogue, and $[\alpha / \mathrm{Fe}]$ 


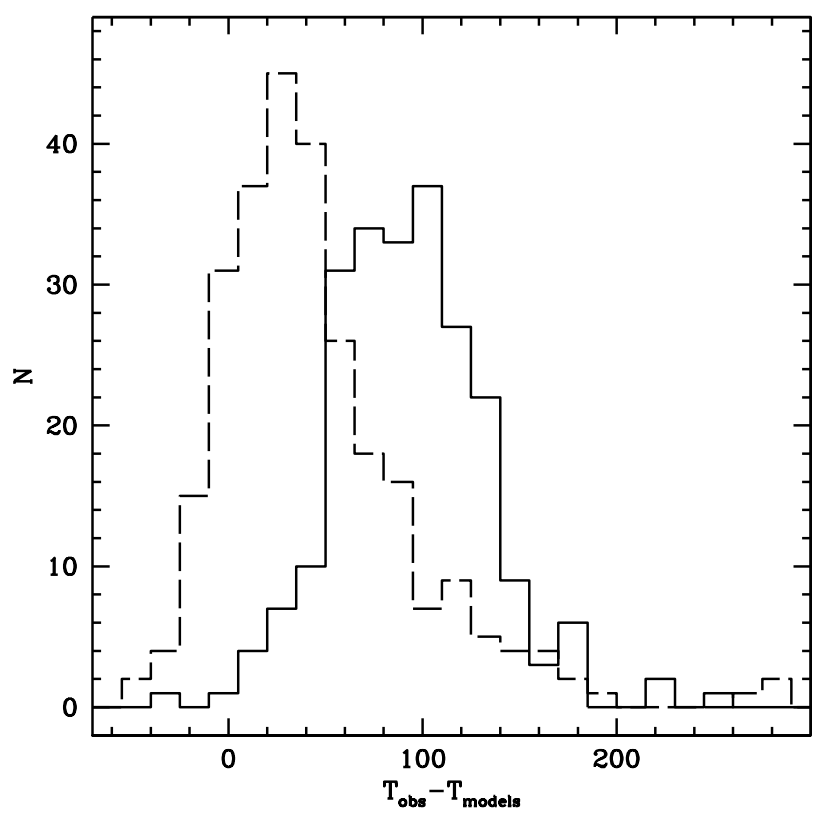

Fig. 6. As Fig. 4 but for the $\Delta T$ values from T17

is actually labelled as $[\alpha / \mathrm{M}]$ in DR13. As explained in the APOGEE catalogue $\mathrm{e}^{3}$, the listed $[\mathrm{M} / \mathrm{H}]$ is an overall scaling of metal abundances assuming a solar abundance ratio pattern, and $[\alpha / \mathrm{M}]=[\alpha / \mathrm{H}]-[\mathrm{M} / \mathrm{H}]_{\odot}$. This definition of $[\mathrm{M} / \mathrm{H}]$ cannot be implemented in stellar evolution calculations in a straightforward fashion for $\alpha$-enhanced metal mixtures, therefore we have extracted from the DR13 catalogue values for $[\mathrm{Fe} / \mathrm{H}]$, and the listed $\alpha$-element abundance ratios $[\mathrm{O} / \mathrm{Fe}],[\mathrm{Mg} / \mathrm{Fe}],[\mathrm{Si} / \mathrm{Fe}]$, $[\mathrm{Ca} / \mathrm{Fe}]$ and $[\mathrm{Ti} / \mathrm{Fe}]$ for all stars in $\mathrm{T} 17$ sample.

The bottom panel of Fig. 7 compares these $[\mathrm{Fe} / \mathrm{H}]$ values with the values listed by $\mathrm{T} 17$ (that correspond to $[\mathrm{M} / \mathrm{H}]$ in DR13). The agreement is typically within \pm 0.02 dex, and suprisingly also for the $\alpha$-enhanced stars. At any rate, the consequence is that the general agreement of the $T_{\text {eff }}$ of our solar $\alpha_{\text {MLT }}$ RGB models with the observed scaled solar metallicy stars is confirmed (as we have verified applying the preocedure described in the previous section, employing these DR13 $[\mathrm{Fe} / \mathrm{H}]$ values) when using the DR13 values labelled as $[\mathrm{Fe} / \mathrm{H}]$.

The top and middle panels of Fig. 7 compare the $[\alpha / \mathrm{Fe}]$ values given by $\mathrm{T} 17$ (that correspond to $[\alpha / \mathrm{M}]$ in DR13 catalogue) with two different estimates of $[\alpha / \mathrm{Fe}]$ based upon the DR13 values of $[\mathrm{O} / \mathrm{Fe}],[\mathrm{Mg} / \mathrm{Fe}],[\mathrm{Si} / \mathrm{Fe}],[\mathrm{Ca} / \mathrm{Fe}]$ and $[\mathrm{Ti} / \mathrm{Fe}]$. In the top panel we display our $[\alpha / \mathrm{Fe}]$ values estimated as $[(\mathrm{Mg}+\mathrm{Si}) / \mathrm{Fe}]$, taking into account that $\mathrm{Mg}$ and $\mathrm{Si}$ are the two $\alpha$-elements that affect the $T_{\text {eff }}$ of RGB stellar evolution models (VandenBerg et al. 2012). We have calculated $[(\mathrm{Mg}+\mathrm{Si}) / \mathrm{Fe}]$ employing the observed $[\mathrm{Mg} / \mathrm{Fe}]$ and $[\mathrm{Si} / \mathrm{Fe}]$, and the Grevesse \& Noels (1993) solar metal mixture used in the model calculations as a reference. The correspondence with $[\alpha / \mathrm{Fe}]$ values listed by T17 (actually $[\alpha / \mathrm{M}]$ in DR13) is remarkable, with an offset of typically just $\sim 0.01$ dex when $[\alpha / \mathrm{Fe}]>0.1$, and a very small spread.

The middle panel displays our $[\alpha / \mathrm{Fe}]$ estimates accounting for all DR13 $\alpha$-elements. On average our $[\alpha / \mathrm{Fe}]$ tend to get systematically larger than T17 values, when $[\alpha / \mathrm{Fe}]$ is larger than $\sim 0.1$ dex, again by just $0.01-0.02$ dex on average. However, there is now a large scatter (compared to the case of $[(\mathrm{Mg}+\mathrm{Si}) / \mathrm{Fe}]$ ) in the differences. The reason for the different behaviour compared to the $[(\mathrm{Mg}+\mathrm{Si}) / \mathrm{Fe}]$ case is that the various $\alpha$ elements

\footnotetext{
http://www.sdss.org/dr12/irspec/aspcap/
}
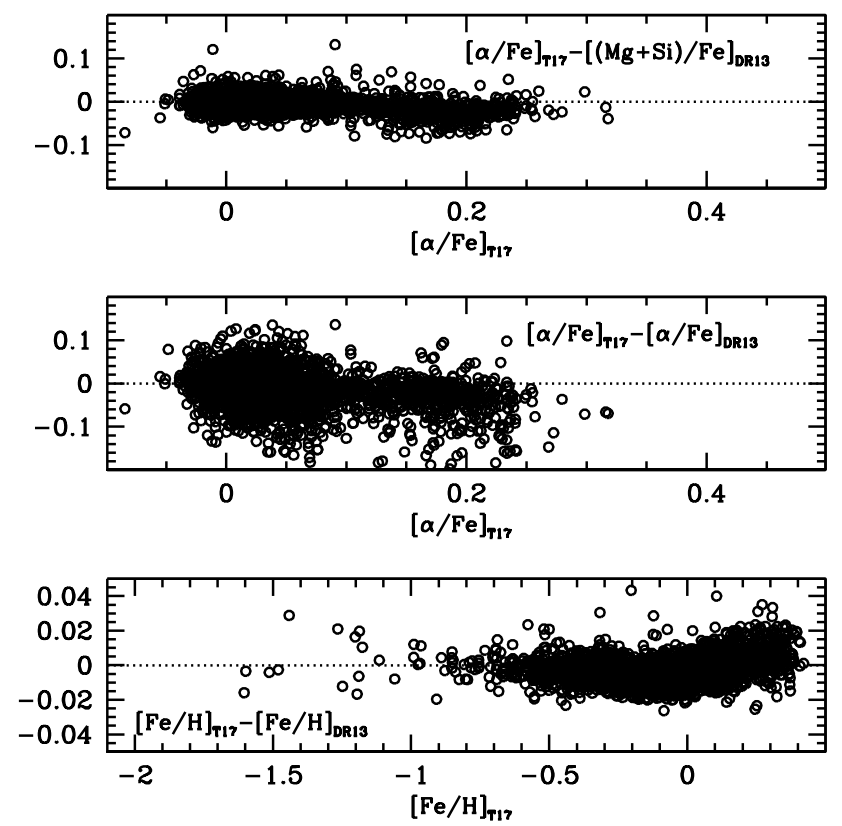

Fig. 7. Bottom panel: difference between $[\mathrm{Fe} / \mathrm{H}]$ values adopted by $\mathrm{T} 17$ $\left([\mathrm{Fe} / \mathrm{H}]_{\mathrm{T} 17}\right)$ and $[\mathrm{Fe} / \mathrm{H}]$ as listed in the DR13 catalogue $\left([\mathrm{Fe} / \mathrm{H}]_{\mathrm{D} 13}\right)$, as a function of $[\mathrm{Fe} / \mathrm{H}]_{\mathrm{T} 17}$. Middle panel: same as bottom panel, but for $[\alpha / \mathrm{Fe}]$, considering the total $\alpha$-enhancement from DR13 data (see text for details). Top panel: same as middle panel, but in this case the DR13 estimate is actually $[(\mathrm{Mg}+\mathrm{Si}) / \mathrm{Fe}]$ (see text for details).

are not always enhanced by the same amount in the observed stars, hence the exact value of $[\alpha / \mathrm{Fe}]$ to some degree depends on which elements are included in its definition.

From the point of view of testing the RGB model $T_{\text {eff }}$, what matters is that the chemical composition of the models match the observed $[(\mathrm{Mg}+\mathrm{Si}) / \mathrm{Fe}]$ (based on the results by VandenBerg et al. 2012). In case of the same enhancement for all $\alpha$ elements, typical of stellar evolution calculations, this means that the model $[\alpha / \mathrm{Fe}]$ has to match the observed $[(\mathrm{Mg}+\mathrm{Si}) / \mathrm{Fe}]$. Therefore the results of the comparison made in the previous section employing T17 $[\alpha / \mathrm{Fe}]$ values still stand, given that $\mathrm{T} 17$ $[\alpha / \mathrm{Fe}]$ corresponds very closely to $[(\mathrm{Mg}+\mathrm{Si}) / \mathrm{Fe}]$ as determined from the DR13 individual abundances.

Finally, Fig. 8 shows very clearly the problem when matching the $T_{\text {eff }}$ of $\alpha$-enhanced stars with models. We have displayed the $\log (g)-T_{\text {eff }}$ diagram of two samples of stars with observed mean mass equal to $1.1 M_{\odot}$ and mean $[\mathrm{Fe} / \mathrm{H}]$ equal -0.35 dex, one with $[\alpha / \mathrm{Fe}]([(\mathrm{Mg}+\mathrm{Si}) / \mathrm{Fe}])$ smaller than $0.07 \operatorname{dex}$ (the scaled solar sample), the other one with average $[\alpha / \mathrm{Fe}]=0.20$ (the $\alpha$-enhanced sample) respectively. These two sets of stars are distributed along well separated sequences, the $\alpha$-enhanced one being redder than the scaled solar sequence, as expected. The $T_{\text {eff }}$ difference between the two sequences is about $110 \mathrm{~K}$ at fixed $\log (g)$

We have also plotted $1.1 M_{\odot},[\mathrm{Fe} / \mathrm{H}]-0.35$ models both scaled solar and with $[\alpha / \mathrm{Fe}]=0.4$, from our own calculations and from Dotter et al. (2008) isochrone database, for a comparison. For these latter models we have used the online webtool ${ }^{4}$ and calculated $[\mathrm{Fe} / \mathrm{H}]=-0.35$ isochrones populated by $\sim 1.1 M_{\odot}$ stars along the RGB.

The observed $T_{\text {eff }}$ difference between scaled solar and $\alpha$ enhanced stars turns out to be reproduced by both independent sets of stellar models for $[\alpha / \mathrm{Fe}] \sim 0.4$, twice the observed value.

4 http://stellar.dartmouth. edu/models/webtools.html 


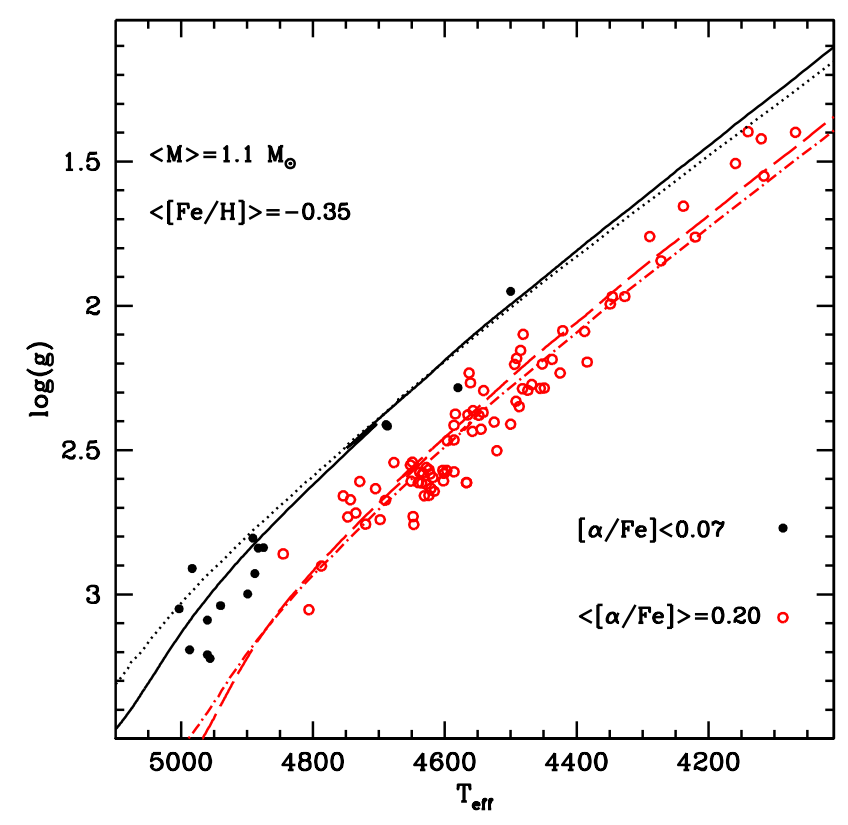

Fig. 8. $\log (g)-T_{\text {eff }}$ diagram of two sub-samples of APOKASC RGB stars with average $[\mathrm{Fe} / \mathrm{H}]=-0.35$ and mass $1.1 M_{\odot}$, but different $\alpha$-enhancements: scaled solar objects $([\alpha / \mathrm{Fe}]<0.07$, filled circles) and $\alpha$-enhanced (average $[\alpha / \mathrm{Fe}]=0.2$, open circles). Our $1.1 M_{\odot},[\mathrm{Fe} / \mathrm{H}]=$ -0.35 evolutionary tracks for $[\alpha / \mathrm{Fe}]=0$ (solid line) and +0.40 (dashed line) are also displayed. The $\alpha$-enhancement of the theoretical models is twice the average enhancement of the selected $\alpha$-enhanced stars. Dotted and dot-dashed lines show tracks with the same mass, $[\mathrm{Fe} / \mathrm{H}]$ and $\alpha$-enhancements, from Dotter et al. (2008).

This further analysis confirms that the trend of $\Delta T$ with $[\mathrm{Fe} / \mathrm{H}]$ obtained with our models is due to the fact that they are systematically hotter than observations for $\alpha$-enhanced stars. Also, this discrepancy between $\alpha$-enhanced RGB stellar models and observations seems to be more general, not just related to our models.

\subsection{The effect of the solar metal distribution}

To assess better the good agreement between our scaled solar models and the RGB sample $([\alpha / \mathrm{Fe}]<0.07)$, we have also calculated a set of models with the same physics inputs but a more recent determination of the solar metal distribution (both opacities and equation of state take into account the new metal mixture), from Caffau et al. (2011) for the most abundant elements, complemented with abundances from Lodders (2010). We have covered the same range of masses and $[\mathrm{Fe} / \mathrm{H}]$ of the reference models employed in the analysis described in the previous sections. Notice that T17 calculations use the Grevesse \& Sauval (1998) solar metal distribution, very similar to the Grevesse \& Noels (1993) one of our reference calculations. The Caffau et al. (2011) solar metal mixture implies a lower metallicity for the Sun compared to Grevesse \& Noels $(1993)^{5}$. Our calibrated solar model provides an initial He abundance $Y=0.269$, metallicity $Z=0.0172, \alpha_{\text {MLT }}=2.0$, and $\Delta Y / \Delta Z=1.31$.

Figure 9 displays the $\Delta T$ values as a function of $[\mathrm{Fe} / \mathrm{H}]$ for the scaled solar sample. We have binned the $\Delta T$ values for $[\mathrm{Fe} / \mathrm{H}]$ larger than $\sim-0.6 \mathrm{dex}(\mathrm{a}[\mathrm{Fe} / \mathrm{H}]$ range of about $1 \mathrm{dex})$, and performed a linear fit to the binned data as in Fig. 2, deriving a slope

\footnotetext{
5 The solar metallicity from the Caffau et al. (2011) determination is slightly higher than what would be obtained with the Asplund et al. (2009) solar metal mixture.
}

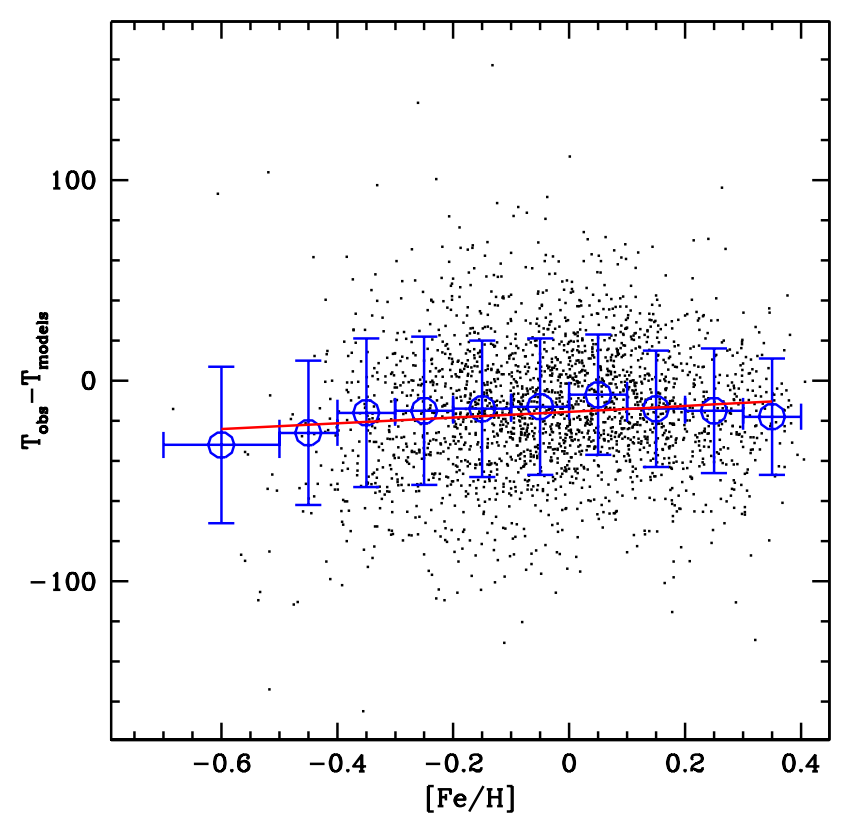

Fig. 9. $\Delta T$ as a function of $[\mathrm{Fe} / \mathrm{H}]$ for the sub-sample of RGB objects with $[\alpha / \mathrm{Fe}]<0.07$, using stellar models calculated with the Caffau et al. (2011) plus Lodders (2010) solar metal distribution (see text for details).

equal to $14 \pm 10 \mathrm{~K} / \mathrm{dex}$, statistically different from zero at much less than $2 \sigma$. The average $\Delta T$ is equal to $-14 \mathrm{~K}$, with a $1 \sigma$ dispersion of $34 \mathrm{~K}$. We can conclude that changing the reference solar metal distribution and the corresponding solar calibrated $\alpha_{\text {MLT }}$ does not alter the agreement between our models and the $T_{\text {eff }}$ of the scaled solar T17 sample of RGB stars.

\subsection{The effect of the model boundary conditions}

As discussed in Kippenhahn et al. (2012), the outer boundary conditions for the solution of the stellar evolution equations have a major effect on models with deep convective envelopes, like the RGB ones. We have therefore explored in some detail this issue, to check whether different choices of how the boundary conditions are determined can cause metallicity dependent $T_{\text {eff }}$ differences amongst models with the same total mass, and eventually - at least partially - explain the differences between our and $\mathrm{T} 17$ results.

The physics inputs of BaSTI and T17 calculations are very similar, the main difference being their integration of the Eddington grey $T(\tau)$ to determine the boundary conditions, whereas we used the VAL $T(\tau)^{6}$. We have therefore investigated the role played by different $T(\tau)$ choices to determine the outer boundary conditions of our model calculations (see also, e.g. Montalbán et al. 2004; VandenBerg et al. 2008, for investigations of the effect of boundary conditions on the $T_{\text {eff }}$ of low-mass stellar models with convective envelopes), when comparing theory with the measured $T_{\text {eff }}$ of T17 sample.

Figure 10 displays two groups of four RGB tracks in the $T_{\text {eff }}-\log (g)$ diagram (within the $T_{\text {eff }}$ and $\log (g)$ range sampled by T17 data), for $1.1 M_{\odot}$ models with the labelled [Fe/H] (scaled solar metal mixtures). The two chosen $[\mathrm{Fe} / \mathrm{H}]$ values bracket

6 The equation of state (EOS) is also different (see T17 and Pietrinferni et al. 2004), but tests made by Pietrinferni et al. (2004) have shown that the EOS employed by T17 produces tracks very close to the ones obtained with the BaSTI EOS choice. 


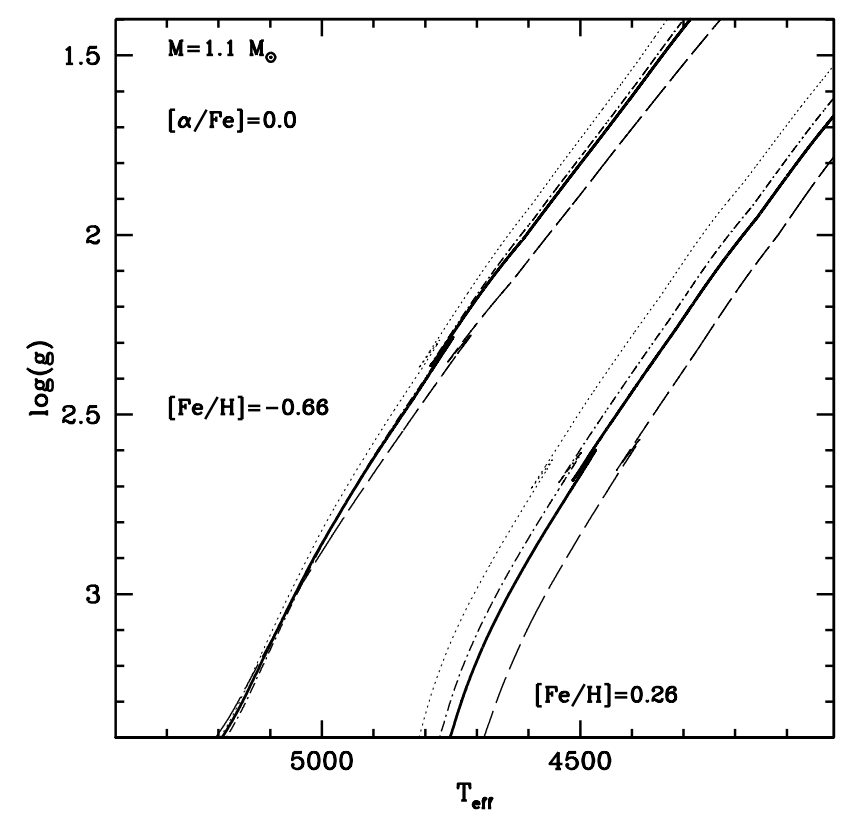

Fig. 10. Scaled solar evolutionary tracks for the labelled mass and $[\mathrm{Fe} / \mathrm{H}]$ values. The tracks correspond to models calculated with the KS (dotted lines), VAL (solid lines), HM (dash-dotted lines) and EDD (dashed lines) $T(\tau)$ relationships, respectively (see text for details).

the metallicity range covered by our $\Delta T-[\mathrm{Fe} / \mathrm{H}]$ analysis, and the four tracks for each $[\mathrm{Fe} / \mathrm{H}]$ represent four different choices for the $T(\tau)$ relation used to determine the outer boundary conditions.

Our reference calculations employing the VAL $T(\tau)$ are plotted together with calculations using an Eddington grey $T(\tau)$ (hereafter EDD) like T17 models, the Krishna Swamy (1966; hereafter KS) and the Holweger \& Mueller (1974; hereafter HM) one ${ }^{7}$. The KS and HM $T(\tau)$ relationships are also solar semi-empirical, like the VAL $T(\tau)$.

Values of $\alpha_{\text {MLT }}$ for these additional models have been fixed again by means of a solar calibration, and are equal to 1.70 (very close to the value 1.72 determined by T17 with their own calculations) 2.11 and 1.99 for calculations with the EDD, KS and HM $T(\tau)$, respectively. For the sake of comparison, we remind the reader that the solar calibration with the VAL $T(\tau)$ requires $\alpha_{\text {MLT }}=1.90$.

It is striking not only that different $T(\tau)$ relations and their corresponding solar calibrated $\alpha_{\mathrm{MLT}}$ values produce RGBs with different $T_{\text {eff }}$ (this was already shown for example in Salaris et al. 2002), but also that differences depend on the model $[\mathrm{Fe} / \mathrm{H}]$. These results are qualitatively and quantitatively the same also for masses equal to 2.0-2.5 $M_{\odot}$, at the upper end of the mass range spanned by T17 data. Clearly, different solar calibrations of $\alpha_{\text {MLT }}$ obtained with different $T(\tau)$ relations do not guarantee consistent behaviours of RGB models with metallicity.

At $[\mathrm{Fe} / \mathrm{H}]=+0.26$, all tracks are roughly parallel. The EDD track is cooler by $\sim 70 \mathrm{~K}$ compared to the reference VAL one, whereas the KS track is hotter by about the same amount, and the HM track is hotter by just $\sim 25 \mathrm{~K}$. At solar metallicity (not shown in the figure) the differences between EDD, VAL, KS and $\mathrm{HM}$ tracks are still about the same as at $[\mathrm{Fe} / \mathrm{H}]=+0.26$, whilst at $[\mathrm{Fe} / \mathrm{H}]=-0.66 \mathrm{EDD}, \mathrm{VAL}$ and $\mathrm{KS}$ tracks are no longer parallel. Above $\log (g) \sim 2.8$ they are roughly coincident, with $T_{\text {eff }}$

7 We employed the analytical fit by VandenBerg \& Poll (1989) to the Holweger \& Mueller (1974) data. differences increasing with decreasing $\log (g)$. At $\log (g)=1.5$ the EDD track is cooler by $\sim 40 \mathrm{~K}$, while the KS track is hotter by $\sim 30 \mathrm{~K}$. The HM track is almost coincident with the VAL one.

We have seen before that $\Delta T$ values determined from our calculations employing the VAL $T(\tau)$ relation do not show any trend with $T_{\text {eff }}$, and are consistent with zero when scaled solar stars are considered. Employing instead the $\operatorname{EDD} T(\tau)$ would increase $\Delta T$ values by $\sim 70 \mathrm{~K}$ at the upper end of the sampled $[\mathrm{Fe} / \mathrm{H}]$ range down to about solar (EDD tracks being systematically cooler than VAL tracks), whilst the increase ranges from negligible to at most $40 \mathrm{~K}$ (when gravity decreases) at the lowest end of the $[\mathrm{Fe} / \mathrm{H}]$ range considered in our analysis. This would induce an overall positive trend in the $\Delta T-[\mathrm{Fe} / \mathrm{H}]$ diagram $(\Delta T$ decreasing with decreasing $[\mathrm{Fe} / \mathrm{H}]$ ) also when restricting the analysis to scaled solar objects, with absolute $\Delta T$ values generally positive. This is at least qualitatively consistent with T17 results, even though it does not fully explain quantitatively T17 results, especially the very large positive $\Delta T$ at solar metallicity. Notice that also the PARSEC calculations - that according to T17 study show also a $\Delta T-[\mathrm{Fe} / \mathrm{H}]$ slope of about $100 \mathrm{~K} / \mathrm{dex}$ - employ an Eddington grey $T(\tau)$ relationship to determine the model outer boundary conditions.

Finally, it is also interesting to notice that Dotter et al. (2008) models display $T_{\text {eff }}$ values very close to ours over the whole mass, surface gravity and $[\mathrm{Fe} / \mathrm{H}]$ range covered by our analysis (see also Fig. 8). In those models the boundary conditions have been taken from a grid of PHOENIX detailed 1D model atmospheres (pressure and temperature at a given optical depth $\tau$, see Dotter et al. 2008) instead of a $T(\tau)$ integration.

\section{Summary and discussion}

Our reanalysis of the T17 sample of RGB stars from the APOKASC catalogue has disclosed the following:

1. According to the APOKASC $T_{\text {eff }}, \log (g)$, mass, $[\mathrm{Fe} / \mathrm{H}]$ and $[\alpha / \mathrm{Fe}]$ values given by T17, theoretical stellar evolution calculations - both our own calculations and T17 models, and also Dotter et al. (2008) calculations - seem to underestimate the effect of $\alpha$-enhancement on the model $T_{\text {eff }}$ at fixed mass, surface gravity and $[\mathrm{Fe} / \mathrm{H}]$.

2. When $\alpha$-enhanced stars are neglected, our RGB models are in good agreement with the empirical $T_{\text {eff }}$ values, with no significant systematic shifts, nor trends with $[\mathrm{Fe} / \mathrm{H}]$, over a $\sim 1$ dex $[\mathrm{Fe} / \mathrm{H}]$ range $([\mathrm{Fe} / \mathrm{H}]$ between $\sim+0.4$ and $\sim-0.6)$. This agreement is preserved also if we change the reference solar metal distribution of our models.

3. For a solar calibrated $\alpha_{\mathrm{MLT}}$, the $T_{\text {eff }}$ differences between theory and observations depend on the choice of the model boundary conditions. It is the combinations of boundary conditions and $\alpha_{\mathrm{MLT}}$ value that determine the $T_{\text {eff }}$ of RGB stellar models, as expected for stars with deep convective envelopes (Kippenhahn et al. 2012).

Regarding the discrepancy between our models and $\alpha$ enhanced stars, a variation of $\alpha_{\mathrm{MLT}}$ with $[\alpha / \mathrm{Fe}]$ at a given $[\mathrm{Fe} / \mathrm{H}]$ seems unlikely - but of course cannot be a-priori dismissed. Another possibility that we have checked is the role played by the $\Delta Y / \Delta Z$ enrichment ratio used in the model calculations. This value is typically fixed by the assumed primordial $\mathrm{He}$ and the solar initial $Y$ (and $Z$ ) obtained from a standard solar model. For a fixed value of $[\mathrm{Fe} / \mathrm{H}], \alpha$-enhanced stars have a larger $Z$, hence the corresponding models will have been calculated with 
a larger $Y$ compared to the scaled solar counterparts at the same $[\mathrm{Fe} / \mathrm{H}]$ (see, e.g. Table 3 in Dotter et al. 2008). What if the initial $Y$ of $\alpha$-enhanced stars is the same as for the scaled solar ones at a given $[\mathrm{Fe} / \mathrm{H}]$ ? In the $[\mathrm{Fe} / \mathrm{H}]$ range of $\mathrm{T} 17$ stars and for the observed $\alpha$-enhancements, the initial $Y$ of the $\alpha$-enhanced models will be at most $\sim 0.01$ larger at the same $[\mathrm{Fe} / \mathrm{H}]$, according to the $\Delta Y / \Delta Z$ value used in our calculations. This small variation of $Y$ increases the $T_{\text {eff }}$ of the models by $\sim 20 \mathrm{~K}$ in the relevant $g$ range, hence our $\alpha$-enhanced models would be at most $20 \mathrm{~K}$ cooler at fixed $[\mathrm{Fe} / \mathrm{H}]$, if $Y$ is the same as for the scaled solar stars. Such a small change of the model $T_{\text {eff }}$ would not erase the discrepancy with observations.

From the empirical point of view, assuming metal abundance, $g$ and $T_{\text {eff }}$ scales are correct, asteroseismic masses systematically too high by $0.1-0.2 M_{\odot}$ for $\alpha$-enhanced stars could explain the discrepancy. Another possibility - assuming mass, $g$ and $T_{\text {eff }}$ values are correct - is that $[\mathrm{Fe} / \mathrm{H}]$ determinations are too low by $\sim 0.1$ dex for each 0.1 dex of $\alpha$-enhancement, or a combination of both mass and $[\mathrm{Fe} / \mathrm{H}]$ systematic errors. Of course it is necessary also to investigate this problem with stellar evolution models, to see whether there is room to explain this discrepancy from the theoretical side.

Concerning possible mixing length variations with chemical composition, we conclude that to match T17 $T_{\text {eff }}$ values for the scaled solar sub-sample, variations of $\alpha_{\mathrm{MLT}}$ with $[\mathrm{Fe} / \mathrm{H}]$ are required only for some choices of the model outer boundary conditions. Depending on the chosen $T(\tau)$ relation, or more in general the chosen set of boundary conditions, a variation of $\alpha_{\mathrm{MLT}}$ with $[\mathrm{Fe} / \mathrm{H}]$ may or may not be necessary. Trying to determine whether $\alpha_{\text {MLT }}$ can be assumed constant irrespective of chemical composition (and mass) for RGB stars thus requires a definitive assessment of the most correct way to determine the model outer boundary conditions.

As a consequence, models calculated with $\alpha_{\mathrm{MLT}}$ calibrations based on 3D radiation hydrodynamics simulations (Trampedach et al. 2014; Magic et al. 2015) are physically consistent only when boundary conditions (and physics inputs) extracted from the same simulations are employed in the stellar model calculations. We have achieved this consistency in Salaris \& Cassisi (2015), testing the impact of Trampedach et al. (2014) simulations on stellar modelling.

Salaris \& Cassisi (2015) have shown that at solar metallicity - the single metallicity covered by these 3D calculations - RGB models calculated with the hydro-calibrated variable $\alpha_{\mathrm{MLT}}$ (that is a function of $T_{\text {eff }}$ and $\log (g)$ ) are consistent - within about $20 \mathrm{~K}$ - with RGB tracks obtained with the solar $\alpha_{\text {MLT }}$ derived from the same set of hydro-simulations. They also found that the VAL $T(\tau)$ relationship provides RGB effective temperatures that agree quite well with results obtained with the hydro-calibrated $T(\tau)$ relationship, within typically $10 \mathrm{~K}$.
Assuming these hydro-simulations are realistic and accurate, the use of the VAL $T(\tau)$ and solar calibrated $\alpha_{\text {MLT }}$ seems to be adequate for RGB stars at solar $[\mathrm{Fe} / \mathrm{H}]$.

The independent 3D hydro-simulations by Magic et al. (2015) cover a large $[\mathrm{Fe} / \mathrm{H}]$ range, from -4.0 to +0.5 , and provide corresponding calibrations of $\alpha_{\mathrm{MLT}}$ in terms of [Fe/H], $T_{\mathrm{eff}}$, and $\log (g)$. However, $T(\tau)$ relationships (or tables of boundary conditions) obtained from their simulations, plus Rosseland mean opacities consistent with the opacities used in their calculations, are not yet available, This means that their $\alpha_{\text {MLT }}$ calibration cannot be consistently implemented in stellar evolution calculations yet, and one cannot yet check consistently whether the variable $\alpha_{\text {MLT }}$ provides RGB $T_{\text {eff }}$ values significantly different from the case a solar hydro-calibrated $\alpha_{\text {MLT }}$ for the full range of $[\mathrm{Fe} / \mathrm{H}]$ covered by these simulations.

Acknowledgements. SC acknowledges financial support from PRIN-INAF2014 (PI: S. Cassisi) and the Economy and Competitiveness Ministry of the Kingdom of Spain (grant AYA2013-42781-P).

\section{References}

Asplund, M., Grevesse, N., Sauval, A. J., \& Scott, P. 2009, ARA\&A, 47, 481 Böhm-Vitense, E. 1958, Z. Astrophys., 46, 108

Bressan, A., Marigo, P., Girardi, L., et al. 2012, MNRAS, 427, 127

Caffau, E., Ludwig, H.-G., Steffen, M., Freytag, B., \& Bonifacio, P. 2011, Sol. Phys., 268, 255

Dotter, A., Chaboyer, B., Jevremović, D., et al. 2008, ApJS, 178, 89

Ferguson, J. W., Alexander, D. R., Allard, F., et al. 2005, ApJ, 623, 585

González Hernández, J. I., \& Bonifacio, P. 2009, A\&A, 497, 497

Gough, D. O., \& Weiss, N. O. 1976, MNRAS, 176, 589

Grevesse, N., \& Noels, A. 1993, Phys. Scr., T47, 133

Grevesse, N., \& Sauval, A. J. 1998, Space Sci. Rev., 85, 161

Holweger, H., \& Mueller, E. A. 1974, Sol. Phys., 39, 19

Iglesias, C. A., \& Rogers, F. J. 1996, ApJ, 464, 943

Kippenhahn, R., Weigert, A., \& Weiss, A. 2012, Stellar Structure and Evolution (Berlin, Heidelberg: Springer-Verlag)

Krishna Swamy K. S. 1966, ApJ, 145, 174

Lodders, K. 2010, Astrophys. Space Sci. Proc., 16, 379

Ludwig, H.-G., Freytag, B., \& Steffen, M. 1999, A\&A, 346, 111

Magic, Z., Weiss, A., \& Asplund, M. 2015, A\&A, 573, A89

Majewski, S. R., Schiavon, R. P., Frinchaboy, P. M., et al. 2017, AJ, 154, 94

Montalbán, J., D’Antona, F., Kupka, F., \& Heiter, U. 2004, A\&A, 416, 1081

Pedersen, B. B., Vandenberg, D. A., \& Irwin, A. W. 1990, ApJ, 352, 279

Pietrinferni, A., Cassisi, S., Salaris, M., \& Castelli, F. 2004, ApJ, 612, 168

Salaris, M., \& Cassisi, S. 1996, A\&A, 305, 858

Salaris, M., \& Cassisi, S. 2008, A\&A, 487, 1075

Salaris, M., \& Cassisi, S. 2015, A\&A, 577, A60

Salaris, M., Cassisi, S., \& Weiss, A. 2002, PASP, 114, 375

Stein, R. F., \& Nordlund, A. 1989, ApJ, 342, L95

Straniero, O., \& Chieffi, A. 1991, ApJS, 76, 525

Tayar, J., Somers, G., Pinsonneault, M. H., et al. 2017, ApJ, 840, 17

Trampedach, R., Stein, R. F., Christensen-Dalsgaard, J., Nordlund, A., \& Asplund, M. 2014, MNRAS, 445, 4366

VandenBerg, D. A., \& Poll, H. E. 1989, AJ, 98, 1451

VandenBerg, D. A., Bolte, M., \& Stetson, P. B. 1996, ARA\&A, 34, 461

VandenBerg, D. A., Edvardsson, B., Eriksson, K., \& Gustafsson, B. 2008, ApJ, 675,746

VandenBerg, D. A., Bergbusch, P. A., Dotter, A., et al. 2012, ApJ, 755, 15

Vernazza, J. E., Avrett, E. H., \& Loeser, R. 1981, ApJS, 45, 635 